\title{
An Output Tracking Integrated Discrete PID-based Sliding Mode Control on SISO Systems
}

\author{
Yu Cao and Xiongbiao Chen \\ The Department of Mechanical Engineering, University of Saskatchewan, Saskatoon S7N 5A9, Canada
}

Received: February 07, 2015 / Accepted: February 28, 2015 / Published: March 25, 2015.

\begin{abstract}
SMC (sliding mode control) has been widely employed to compensate for the system uncertainty and disturbance. However, the chattering problem, caused by the discontinuous characteristic of switching function used in traditional SMC, greatly deteriorates the performance of SMC and has become the main limitation for its applications. Also, implementing the SMC in digital systems could make it even worse due to the limited sampling time. Moreover, as a state tracking control scheme, traditional SMC cannot be employed in the applications where the system states are not available. To alleviate these problems, the paper presents the development of a novel control method, so called "the output tracking integrated discrete PID (proportional-integral-derivative)-based SMC" for the SISO (single-input-single-output) system, along with the controller design approaches (i.e., the traditional SMC design approach and the model reference approach). Without the need of system states, this novel method allows for eliminating chattering problem and the steady state error that may exists in such control methods as the continuous PID-based SMC. In order to demonstrate the effectiveness of the developed method, experiments were carried out on a commercially available piezoelectric actuator with varying sampling times, as compared to the continuous PID-based SMC. The results illustrate that the tracking performance with the proposed method is much better than the continuous PID-based SMC.
\end{abstract}

Key words: Discrete, PID, SISO, sliding mode control.

\section{Introduction}

SMC (sliding model control) is a form of variable structure control and has been recently drawing considerable attention in the control research community worldwide due to its ability to compensate for the system uncertainties and disturbance [1-4]. However, because of the discontinuity of SMC switching control, chattering exists and thus excites undesired system high resonance mode to deteriorate the system tracking performance. One solution to the chattering problem is to use the boundary layer control [1], in which a saturation switching control is employed to replace the discontinuous switching control. It is noted that, if the unknown disturbance is profound, a sufficiently-high gain in controller is always required and such a control scheme behaves

Corresponding author: Xiongbiao Chen, professor, Ph.D., research fields: mechanical engineering and bio-medical engineering. E-mail: xbc719@mail.usask.ca. like a high-gain proportional (P) controller. As a result, steady state error may exist. An alternative way is to enlarge the width of the boundary layer, thus decreasing the effective linear gain for reduced state oscillation around sliding surface. However the state no longer strictly locates on the ideal sliding surface due to the wider boundary layer and the system does not behave as described by the sliding mode. In Ref. [5], a high order sliding surface was used to replace the first order one typically used in the nominal SMC design. With the main advantages of the nominal SMC, the HOSMC (high order sliding mode control) can reduce the chattering effect and improve the accuracy for its realization. The main problem is that it requires increasing sliding information in implementing the HOSMC [6]. Recently, a new continuous PID (proportional-integral-derivative)-based SMC was developed [7], in which the discontinuous 'bang-bang' switching function is replaced by a PID regulator in 
order to eliminate the chattering problem. Due to the integral effect of the PID regulator, the steady state tracking error can be eliminated.

As a state tracking control scheme, the PID-based SMC developed in Ref. [7] has been shown effective in the control of the second order mechanical systems, in which the states can be readily estimated from the measured output based on its physical model. In some applications, the system states may not be readily, or even be impossibly, obtained [8] due the system complexity. In such cases, the application of the PID-based is challenged due to the lack of information of system states [2].

Furthermore, it is noticed that, if implemented in a digital computer, the continuous SMC (CSMC) may not work as expected [9, 10]. For discrete SMC (DSMC), if the switching time does not match the sampling time, the states will not lay on the sliding surface and the trajectory appears like a zigzag motion around the sliding surface, which suggests that the sampling itself also induces the chattering problem in the DSMC. If the mean of zigzag motion deviates from the sliding surface, the steady state error will exist. Therefore, the appropriate forms of discrete SMC are desirable to alleviate the aforementioned problems, especially for the applications where the sampling rate is limited. In Ref. [11], a robust DSMC design was proposed for a nonlinear electrical hydraulic actuator system; and the state required was measured by means of a position sensor. In Ref. [12], the DSMC design was combined with a state estimator and disturbance observer, which, however, significantly increased the system complexity.

To raise these challenges, this paper presents the development of a novel control method, so called "the output tracking based DSMC", in which the system output is integrated in the SMC design such that the proposed method can be employed in the applications where the system states are not available. In addition, there are sensors or devices that may directly give the corresponding measurements of output position, velocity and acceleration and therefore, application of the output tracking based DSMC economizes the costs in the plant and controller implementation. Furthermore, the proposed method is cascaded with the optimal inversion control to further improve the system dynamics, in which the error caused by the imperfect compensation of the optimal inversion is treated as a disturbance and then rejected by the PID-based SMC. In addition, due to the application of the PID regulator in the proposed method, as designed by means of the traditional SMC design approach and the model reference approach, the state zigzag motion, as observed in other DSMCs, is eliminated for improved performance. In order to verify the effectiveness of the proposed method, experiments were carried out on a commercially available PEA (piezoelectric actuator), as compared to the continuous PID-based SMC.

\section{Output Tracking Integrated PID-based Sliding Mode Control}

\subsection{Problem Statement}

Consider a discrete nth order SISO system with the following transfer function

$$
G(z)=\sum_{i=0}^{n} \beta_{i} z^{-i} /\left(1+\sum_{i=1}^{n} \alpha_{i} z^{-i}\right)
$$

where, $\quad \alpha_{i}, \beta_{i}(i=1,2, \cdots, n) \quad$ and $\quad \beta_{0} \quad$ are the parameters and the numerator and denominator are relatively prime polynomials. If $\beta_{i}=0$ for $i=1,2, \cdots, n$, the discrete transfer function can be rewritten in a state space form.

$$
x(k+1)=A_{d} x(k)+B_{d} v(k)
$$

where, $x(k)=\left[\begin{array}{lll}y(k-n) & \cdots & y(k-1)\end{array}\right]^{T}$ is the state vector; $v(k)$ is the control input signal vector

$$
\begin{aligned}
& A_{d}=\left[\begin{array}{cc}
0_{(n-1) \times 1} & I_{(n-1) \times(n-1)} \\
-\alpha_{n} & \boldsymbol{\alpha}
\end{array}\right] \\
& \left(\boldsymbol{\alpha}=\left[\begin{array}{llll}
-\alpha_{n-1} & -\alpha_{n-2} & \cdots & -\alpha_{1}
\end{array}\right]\right) \\
& B_{d}=\left[\begin{array}{ll}
0_{1 \times(n-1)} & \beta_{0}
\end{array}\right]^{T}
\end{aligned}
$$


are the system matrixes of the discrete state space model. The state vector is represented in terms of the outputs in the past history. As such, the method introduced in Ref. [7] can be adopted and applied in the design SMC.

For the systems whose model is obtained by means of system identification with $\beta_{i} \neq 0 \quad(i=1,2, \cdots, n)$, the aforementioned state space form does not exist and as such, an output tracking based design process has to be employed. For this, the transfer function (1) is regarded as the cascade of the nominator $G_{1}(z)=\sum_{j=0}^{n} \beta_{i} z^{-j} / \beta_{0}$ and $G_{2}(z)=\beta_{0} /\left(1+\sum_{i=1}^{n} \alpha_{i} z^{-i}\right)$.

It is noted that $G_{2}(z)$ can be expressed by such a state space model, as shown in Eq. (2). Therefore, the output of $G_{1}(z)$ or the input to $G_{2}(z)$, dented by $v(z)$ in the following, can be derived from the traditional SMC design. The input $u$ can be obtained by cascading the inverse of the transfer function $G_{1}(z)$, denoted by $G_{1}^{-1}(z)$, to the discrete PID-based SMC which is designed based on $G_{2}(z)$. It is noted that, due to the imperfection of the plant model, the dynamics of $G_{1}(z)$ may not be completely inverted by the use of $G_{1}^{-1}(z)$. Such an error due to the model imperfection is considered as an uncertainty added to the input of the plant and will be compensated by the PID-based SMC, as shown in Fig. 1.

\subsection{Traditional Design}

Consider Eq. (2) with the bounded and matched uncertainties and disturbance

$$
x(k+1)=A_{d} x(k)+B_{d} v(k)+\varepsilon(k)
$$

Denote $x_{d}(k)=\left[\begin{array}{lll}y_{d}(k-n) & \cdots & y_{d}(k-1)\end{array}\right]^{T}$. The objective of DSMC is to force the error state

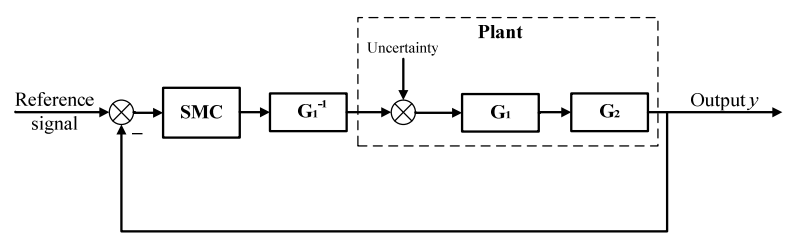

Fig. 1 Inversion based design approach of DSMC for the SISO plant. $e(k)=x(k)-x_{d}(k)$, staring from any initial value, to move to the sliding surface and then converge to zero. Equation (3) can be rewritten in terms of the dynamics of $e(k)$,

$$
\begin{aligned}
e(k+1) & =A_{d} e(k)+B_{d} v(k)+\varepsilon(k) \\
& +A_{d} x_{d}(k)-x_{d}(k+1)
\end{aligned}
$$

Similar to the general design approach for the continuous system, the input $v(k)$ can be divided into two parts $v_{1}(k)$ and $v_{S M}(k)$, i.e.,

$$
v_{1}(k)=-B_{d}^{\dagger}\left[A_{d} x_{d}(k)-x_{d}(k+1)\right]
$$

where, $B_{d}^{\dagger}$ is the pseudo inverse of matrix $B_{d}$.

Substituting Eq. (5) into Eq. (4) yields

$$
\begin{aligned}
& e(k+1)=A_{d} e(k)+B_{d} v_{S M}+\varepsilon(k) \\
& +\left(I-B_{d} B_{d}^{\dagger}\right) A_{d} x_{d}(k)-\left(I-B_{d} B_{d}^{\dagger}\right) x_{d}(k+1)
\end{aligned}
$$

For the system described by Eq. (3), it can be $\operatorname{verified}\left(I-B_{d} B_{d}^{\dagger}\right) A_{d} x_{d}(k)-\left(I-B_{d} B_{d}^{\dagger}\right) x_{d}(k+1)=0$.

Thus, Eq. (6) is reduced to

$$
e(k+1)=A_{d} e(k)+B_{d} v_{S M}+\varepsilon(k)
$$

For the sliding function that takes the following form of

$$
s(k)=\operatorname{Se}(k)
$$

where, $S$ defines the shape of the sliding surface, the control action can be considered consisting two parts, i.e.,

$$
v_{S M}(k)=v_{e q}(k)+\Delta v(k)
$$

where, $v_{e q}(k)=-\left(S B_{d}\right)^{-1} S A_{d} e(k)$ is the equivalent control and $\Delta v(k)$ is the switching control. Substituting Eq. (9) into Eqs. (7) and (8) yields

$$
\begin{aligned}
e(k+1) & =\left[I-B_{d}\left(S B_{d}\right)^{-1} S\right] A_{d} e(k) \\
& +B_{d} \Delta v(k)+\varepsilon(k) \\
& s(k+1)=S B_{d} \Delta v(k)+S \varepsilon(k)
\end{aligned}
$$

For the convenience of following discussion, Eqs. (8) and (10) are rewritten as: 


$$
\begin{aligned}
e(k+1) & =A_{p} e(k)+B_{d} \Delta v(k)+\varepsilon(k), \\
s(k) & =\operatorname{Se}(k)
\end{aligned}
$$

where, $A_{p}=\left[I-B_{d}\left(S B_{d}\right)^{-1} S\right] A_{d}$. The system described by Eq. (12) is equivalent to a dynamic plant, where $\Delta v(k)$ is the input and $s(k)$ is the output. The aim of the controller design is to force the output of the equivalent plant to be zero. Instead of using the switching control, a PID regulator is employed to generate the control signal in this paper, as shown in Fig. 2, which is given by

$$
\Delta v(k)=-\left[P s(k)+I \sum_{i=0}^{k} s(i) T+D \frac{s(k)-s(k-1)}{T}\right]
$$

where $P, I$ and $D$ are parameters of the discrete PID-based SMC; $T$ is the sampling period.

Theorem 1: If the closed-loop system is stable, by using the PID regulator stated in Eq. (13), the steady state output of the equivalent plant (12) will be zero [13], i.e., $s(\infty)=\lim _{z \rightarrow 1} s(z)=0$.

Theorem 2: There exist such parameters $P, I$ and $D$ that the closed-loop control system in Fig. 2 is stable [14].

The input $u$ can be obtained by an inverse feedforward control, as shown in Fig. 1.Taking the inverse Z-Transform of $G_{1}(z)$ yields:

$$
u(k)=\left[\beta_{0} v(k)-\beta_{1} u(k-1)-\cdots-\beta_{n} u(k-n)\right] / \beta_{0}(14)
$$

This recursive equation can be readily implemented in digital systems. It is noted in Eq. (14) that the poles in the transfer function from $v(z)$ to $u(z)$ are the zeros of the plant (1). Therefore, the stability of the traditional SMC design approach depends on the location of the zeros of the plant. For the SISO system with zeros locating outside the unit circle, the divergent input $u$ needs to be generated from Eq. (14). To solve this problem, the optimal inversion, as reported in Ref. [15], is adopted and used in the present paper. The objective of the optimal inversion is to develop an input $u$ such that the following cost function is minimized

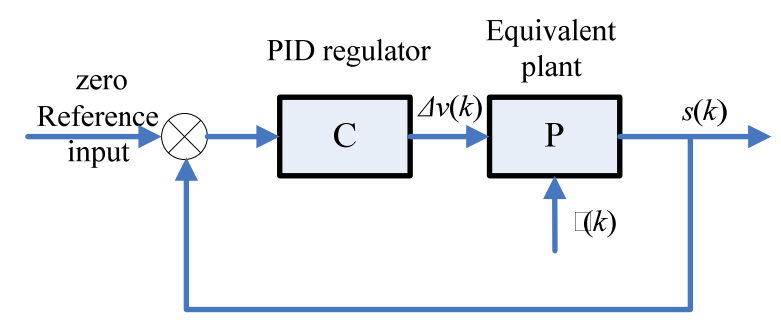

Fig. 2 Equivalent plant for the design of $\Delta v(k)$.

$$
\begin{aligned}
J & =\left[u(k)-u_{d}(k)\right]^{T} Q\left[u(k)-u_{d}(k)\right] \\
& +u^{T}(k) R u(k)
\end{aligned}
$$

where, $u_{d}(k)=\left[\beta_{0} v(k)-\cdots-\beta_{n} u(k-n)\right] / \beta_{0}$,

$Q$ and $R$ are weight matrixes, which determine the stability of the optimal inversion. As such, the optimal input is given by

$$
u_{\text {opt }}(k)=\frac{Q\left[\beta_{0} v(k)-\cdots-\beta_{n} u(k-n)\right]}{\beta_{0}(R+Q)}
$$

\subsection{Model Reference Design of the Output Tracking Based DSMC}

An alternative way to design the output tracking based DSMC is the model reference approach, whose objective is to develop a control action which forces the plant dynamics to follow the dynamics of an ideal model. The controller should thus, force the error between the actual output $y$ and the desired output calculated by the reference model $y_{d}$ to zero as time approaches to infinity.

For the $n$th order discrete SISO system that is described by Eq. (1) with bounded disturbance and uncertainties, the state space representation is

$$
\begin{aligned}
& x(k+1)=A_{d} x(k)+B_{d} u(k)+\varepsilon(k) \\
& y(k)=C_{d} x(k)+D_{d} u(k)
\end{aligned}
$$

It is noted that $D_{d}$ does not have to be a zero matrix. Suppose the desired state space representation is

$$
\begin{aligned}
& w(k+1)=A_{m} w(k)+B_{m} r(k) \\
& y_{d}(k)=C_{m} w(k)+D_{m} r(k)
\end{aligned}
$$

where, $w(k) \in R^{m \times 1}$ is the state; $r(k) \in R$ is the reference input signal; $y_{d}(k) \in R$ is the output of the 
reference system; $A_{m} \in R^{m \times m} \quad, \quad B_{m} \in R^{m \times 1}$, $C_{m} \in R^{1 \times m}$ and $D_{m} \in R$ are the system matrixes.

Denoting the tracking error as:

$$
e(k)=y(k)-y_{d}(k)
$$

With the denotation of

$$
\begin{aligned}
\delta(k) & =[e(k), \cdots, e(k+n-1)]^{T}, \text { Eq. (19) leads to, } \\
\delta(k) & =A_{c} x(k)+B_{c 0} u(k)+\cdots+B_{c(n-1)} u(k+n-1) \\
& +\Delta(k)-A_{c m} w(k)-B_{c m 0} r(k) \\
& -\cdots-B_{c m(n-1)} r(k+n-1)
\end{aligned}
$$

where, $\Delta(k)$ represents the effective disturbance and uncertainty of the system,

$$
\begin{aligned}
& A_{c}=\left[\begin{array}{lllll}
C_{d}{ }^{T} & \left(C_{d} A_{d}\right)^{T} & \left(C_{d} A_{d}{ }^{2}\right)^{T} & \cdots & \left(C_{d} A_{d}{ }^{n-1}\right)^{T}
\end{array}\right]^{T} ; \\
& B_{c 0}=\left[\begin{array}{lllll}
D_{d}{ }^{T} & \left(C_{d} B_{d}\right)^{T} & \left(C_{d} A_{d} B_{d}\right)^{T} & \cdots & \left(C_{d} A_{d}{ }^{n-2} B_{d}\right)^{T}
\end{array}\right]^{T} \\
& B_{c 0}=\left[\begin{array}{lllll}
0 & D_{d} & \left(C_{d} B_{d}\right)^{T} \quad \cdots \quad\left(C_{d} A_{d}^{n-3} B_{d}\right)^{T}
\end{array}\right]^{T} \cdots \\
& B_{c(n-1)}=\left[\begin{array}{lllll}
0 & 0 & 0 & \cdots & D_{d}
\end{array}\right]^{T} \text {, } \\
& B_{c m 1}=\left[\begin{array}{lllll}
0 & D_{m} & \left(C_{m} B_{m}\right)^{T} \quad \cdots \quad\left(C_{m} A_{m}^{n-3} B_{m}\right)^{T}
\end{array}\right]^{T} \cdots \\
& B_{c m(n-1)}=\left[\begin{array}{lllll}
0 & 0 & 0 & \cdots & D_{m}
\end{array}\right]^{T} \text {, } \\
& A_{c m}=\left[\begin{array}{lllll}
C_{m}{ }^{T} & \left(C_{m} A_{m}\right)^{T} & \left(C_{m} A_{m}{ }^{2}\right)^{T} & \cdots & \left(C_{m} A_{m}{ }^{n-1}\right)^{T}
\end{array}\right]^{T} ; \\
& B_{c m 0}=\left[\begin{array}{lllll}
D_{m}^{T} & \left(C_{m} B_{m}\right)^{T} & \left(C_{m} A_{m} B_{m}\right)^{T} & \cdots & \left(C_{m} A_{m}{ }^{n-2} B_{m}\right)^{T}
\end{array}\right]^{T} \\
& \Delta(k)=\left[\begin{array}{lllll}
0_{n \times 1} & \left(C_{d}\right)^{T} & \left(C_{d} A_{d}\right)^{T} & \cdots & \left(C_{d} A_{d}^{n-2}\right)^{T}
\end{array}\right]^{T} \varepsilon(k) \\
& +\left[\begin{array}{lllll}
0_{n \times 1} & 0_{n \times 1} & C_{d}^{T} & \cdots & \left(C_{d} A_{d}^{n-3}\right)^{T}
\end{array}\right]^{T} \varepsilon(k+1) \\
& +\cdots+\left[\begin{array}{lllll}
0_{n \times 1} & 0_{n \times 1} & 0_{n \times 1} & \cdots & 1_{n \times 1}
\end{array}\right]^{T} \varepsilon(k+n-2),
\end{aligned}
$$

Eq. (20) leads to

$$
\begin{aligned}
\delta(k+1) & =A_{c} A_{d} x(k)+A_{c} B_{d} u(k)+B_{c 0} u(k+1) \\
& +\cdots+B_{c(n-1)} u(k+n)+A_{c} \varepsilon(k) \\
& +\Delta(k+1)-A_{c m} A_{m} w(k)-A_{c m} B_{m} r(k) \\
& -B_{c m 0} r(k+1)-\cdots-B_{c m(n-1)} r(k+n)
\end{aligned}
$$

It is noted that $A_{c} \in R^{n \times n}$. Under the assumption that system (21) be observable, i.e., $\left|A_{c}\right| \neq 0$, one has $\delta(k+1)=A_{e} \delta(k)+\Gamma_{0} u(k)+\cdots+\Gamma_{n-1} u(k+n-1)+\bar{\Delta}(k)$ $+B_{c(n-1)} u(k+n)+P w(k)+R_{0} r(k)+\cdots+R_{n} r(k+n)$ where, $A_{e}=A_{c} A_{d} A_{c}^{-1} \delta(k), P=A_{c} A_{d} A_{c}^{-1} A_{c m}-A_{c m} A_{m}$, $\Gamma_{0}=A_{c} B_{d}-A_{c} A_{d} A_{c}^{-1} B_{c 0}, \Gamma_{n-1}=B_{c(n-2)}-A_{c} A_{d} A_{c}^{-1} B_{c(n-1)}$, $\bar{\Delta}(k)=A_{c} \varepsilon(k)+\Delta(k+1)-A_{c} A_{d} A_{c}^{-1} \Delta(k)$, $R_{0}=A_{c} A_{d} A_{c}^{-1} B_{c m 0}-A_{c m} B_{m}, \quad R_{1}=A_{c} A_{d} A_{c}^{-1} B_{c m 1}-B_{c m 0}$, $\ldots, R_{n-1}=A_{c} A_{d} A_{c}^{-1} B_{c m(n-1)}-B_{c m(n-2)}, R_{n}=-B_{c m(n-1)}$.

Eq. (22) has similar form as Eq. (4). As such the traditional SMC design can be employed to generate the control signal $u$. Following the design as shown in Eq. (5), one has the control signal consisting of two parts $u(k)=u_{1}(k)+u_{2}(k)$. Therefore, Eq. (22) can be re-written as:

$$
\begin{aligned}
\delta(k+1) & =A_{e} \delta(k)+P w(k)+f\left(u_{1}\right) \\
& +f\left(u_{2}\right)+\bar{\Delta}(k)+g(r)
\end{aligned}
$$

where,

$$
\begin{gathered}
f\left(u_{1}\right)=\Gamma_{0} u_{1}(k)+\cdots+\Gamma_{n-1} u_{1}(k+n-1)+B_{c(n-1)} u_{1}(k+n) \\
f\left(u_{2}\right)=\Gamma_{0} u_{2}(k)+\cdots+\Gamma_{n-1} u_{2}(k+n-1)+B_{c(n-1)} u_{2}(k+n) \\
g(r)=R_{0} r(k)+R_{1} r(k+1)+\cdots+R_{n} r(k+n) . \text { If } \\
P w(k)+f\left(u_{1}\right)+g(r)=0
\end{gathered}
$$

then Eq. (23) leads to a nominal SMC problem.

2.3.1 Determination of $u_{1}$

From Eq. (24), one has:

$$
\begin{aligned}
& u_{1}(k+n)=-B_{c(n-1)}^{\dagger}\left[P w(k)+\Gamma_{0} u_{1}(k)+\cdots\right. \\
& \left.+\Gamma_{n-1} u_{1}(k+n-1)+R_{0} r(k)+\cdots+R_{n} r(k+n)\right]
\end{aligned}
$$

Substituting Eq. (25) into Eqs. (23) and (24),

$\left(I-B_{c(n-1)} B_{c(n-1)}^{\dagger}\right) P w(k)+\left(I-B_{c(n-1)} B_{c(n-1)}^{\dagger}\right) \Gamma_{0} u_{1}(k)+\cdots$ $+\left(I-B_{c(n-1)} B_{c(n-1)}^{\dagger}\right) \Gamma_{n-1} u_{1}(k+n-1)+\left(I-B_{c(n-1)} B_{c(n-1)}^{\dagger}\right)$ - $R_{0} r(k)+\cdots+\left(I-B_{c(n-1)} B_{c(n-1)}^{\dagger}\right) R_{n} r(k+n)=0$

Eq. (26) is satisfied for any $w$ and $r$ if and only if $\left(I-B_{c(n-1)} B_{c(n-1)}^{\dagger}\right) P=0,\left(I-B_{c(n-1)} B_{c(n-1)}^{\dagger}\right) \Gamma_{0}=0, \cdots$, $\left(I-B_{c(n-1)} B_{c(n-1)}^{\dagger}\right) \Gamma_{n-1}=0,\left(I-B_{c(n-1)} B_{c(n-1)}^{\dagger}\right) R_{0}=0, \cdots$, $\left(I-B_{c(n-1)} B_{c(n-1)}^{\dagger}\right) R_{n}=0$

It can be verified that for a controllable and observable SISO system described by Eq. (1), the conditions as given in (27) are satisfied if the reference 
model is selected such that $C_{m} A_{m}^{i-1} \neq 0_{1 \times m}$ $(i=1,2, \cdots, n)$. This indicates that the model reference approach for the discrete SISO system can be transferred to the nominal DSMC design.

\subsubsection{Determination of $u_{2}$}

Let the sliding surface be $s(k)=S \delta(k)=0$, following the nominal SMC design as shown in Eqs. (7)-(9), one has the control action consisting of two parts, i.e., $u_{2}(k)=u_{e q}(k)+\Delta u(k)$, where,

$$
\begin{gathered}
u_{e q}(k+n)=-\left(S B_{c(n-1)}\right)^{-1} S\left[A_{e} \delta(k)+\Gamma_{0} u_{2}(k)\right. \\
\left.+\cdots+\Gamma_{n-1} u_{2}(k+n-1)\right]
\end{gathered}
$$

and the PID regulator can be given by

$$
\Delta u(k)=-\left[P s(k)+I \sum_{i=0}^{k} s(i) T+D \frac{s(k)-s(k-1)}{T}\right]
$$

\subsubsection{Stability Analysis}

It is noticed that $w(k)$ is a function of $r(k), r(k+$ 1), $\quad$.., $\quad r(k+n), \quad+\quad$ thus $g[r(k), \cdots, r(k+n)]=d u_{1}(k+n)+$ $\left(b_{0}-a_{0} d\right) u_{1}(k)+\cdots+\left(b_{n-1}-a_{n-1} d\right) u_{1}(k+n-1)$ $=P w(k)+R_{0} r(k)+\cdots+R_{n} r(k+n)$. By taking $\mathrm{Z}$-Transform, the transfer function from $r$ to $u_{1}$ is:

$$
\frac{u_{1}(z)}{r(z)}=\frac{g(z)}{d z^{n}+\left(b_{n-1}-a_{n-1} d\right) z^{n-1}+\cdots+\left(b_{0}-a_{0} d\right)}
$$

Eq. (30) indicates that the poles of the transfer function from $r$ to $u_{1}$ are the zeros of the plant transfer function. Therefore, location of the zeros of the plant determines the stability in the model reference approach. Particularly, if the zeros of the plant are located outside the unit circle in the complex plane, optimal inversion (16) can also be applied.

\section{Experiment and Results}

PEAs have been widely used in nanotechnology due to its high precision, fast response and large force generated. However, hysteresis, existing in PEA as a nonlinear effect, can greatly degrade their performance [16]. To improve the performance of PEAs, control and compensation for hysteresis have been drawing considerable attention. In this paper, with the aim of verifying the effectiveness of the discrete PID-based SMC, experiments were designed and carried out on a commercially- available PEA (P-753, Physik Instrumente). The hysteresis nonlinearity existing in the PEA was considered as a disturbance, which was rejected by the proposed discrete PID-based SMC.

The PEA selected for experiments can generate displacement in a range of $15 \mu \mathrm{m}$ with a resolution of $0.5 \mathrm{~nm}$. For displacement measurements, a built-in capacitive displacement sensor with a resolution of 1 $\mathrm{nm}$ was used. Both the actuator and the sensor were connected to a host computer via an $\mathrm{I} / \mathrm{O}$ board (PCI-DAS1602/16, Measurement Computing Corporation) and controlled via SIMULINK programs. The mass ratio of the stage driven by the PEA to the PEA is 49.8, suggesting that the dynamics of the whole system can be regarded approximately as a second order system [17, 18]. As such, a second order auto-regressive model was chosen to describe the system dynamics, while the hysteresis exhibited by the actuator is considered as an extra disturbance to the dynamic model. As a result, sophisticated models for hysteresis are not required in the implementation of developed control. For the experiments, a $70 \mathrm{~V}$ white noise input voltage was provided to the PEA and the corresponding output displacements were measured at sampling rate of $1,000 \mathrm{~Hz}, 2,000 \mathrm{~Hz}, 5,000 \mathrm{~Hz}, 10,000$ $\mathrm{Hz}$, and $20,000 \mathrm{~Hz}$, respectively. With the recorded data, the parameters were identified by using the least square method. The identified results shows that $\beta_{1}$ and $\beta_{2}$ in Eq. (1) are not zero, which indicates that the traditional state tracking based SMC as shown in Ref. [7] are not applicable in this situation.

\subsection{Output Tracking Integrated Discrete PID-based SMC-Traditional SMC Design Approach}

The output tracking integrated discrete PID-based SMC developed by using the traditional SMC design approach was implemented in the experiments to 
control the PEA with different sampling frequencies $1,000 \mathrm{~Hz}$ and $20,000 \mathrm{~Hz}$. A $5 \mu \mathrm{m}$ step input was provided as the reference signal. The state was designed to be $x=[y ; v]$, where $y$ is the output displacement and $v$ is the velocity. The displacement and the velocity were estimated by a $\alpha-\beta$ filter which is a simplified observer for estimation and filtering. The parameters of the filter was adjust by trails-and-errors as $\alpha=0.1, \beta=0.0001$ for $20,000 \mathrm{~Hz}$ sampling rate and $\alpha=0.3, \beta=0.001$ for $1,000 \mathrm{~Hz}$ sampling rate. Matrix $S$ in Eq. (8) was defined as $S=[m, 1]$. Since the zeros of the plant locate outside the unit circle, the optimal stable inversion (16) was integrated in the controller. For comparison, a nominal output tracking based DSMC was also implemented to control the PEA. The sliding surface was designed the same as the discrete PID-based SMC. 'Bang-bang' control was applied as the switching control. The results show that serious chattering problem exists in the nominal output tracking based DSMC with 'bang-bang' switching control. In addition, the steady state error exists in the nominal DSMC.

By using the PID-based switching control instead of the 'bang-bang' switching, chattering problem and steady state error were eliminated. The error state converges to zero and oscillates around the origin point with its amplitude being $0.015 \mu \mathrm{m}$ at $20,000 \mathrm{~Hz}$ sampling rate and $0.006 \mu \mathrm{m}$ at $1,000 \mathrm{~Hz}$ sampling rate, which are the noise level of the sensor.

\subsection{Output Tracking Integrated Discrete PID-based SMC-Model Reference Approach}

In this section, the output tracking integrated discrete PID-based SMC was designed by using the model reference approach. Experiments with both $1,000 \mathrm{~Hz}$ and $20,000 \mathrm{~Hz}$ sampling rates were carried out. A $5 \mu \mathrm{m}$ step input was adopted as the reference signal as well. The state and the $\alpha-\beta$ filter were defined the same as the traditional SMC design approach. The transfer function of the desired second order system was given by $G_{d}(s)=\omega_{n}^{2} /\left(s^{2}+2 \varsigma \omega_{n} s+\omega_{n}^{2}\right)$ where $\varsigma$ is the desired damping ratio and $\omega_{n}$ is the desired natural frequency. With $\varsigma$ and $\omega_{n}$ being chosen as 0.7 and $1,000 \mathrm{rad} / \mathrm{s}$, respectively, a step response with $5.7 \mathrm{~ms}$ setting time and $4.6 \%$ overshoot can be obtained. The optimal stable inversion (16) was additionally integrated in the controller. Parameters in the proposed method designed by the model reference approach were adjusted such that the best tracking performance can be achieved. The results show that the setting time and the overshoot were respectively $5.5 \mathrm{~ms}$ and $5.2 \%$ for the $20,000 \mathrm{~Hz}$ sampling rate and $4.2 \mathrm{~ms}$ and $4.3 \%$ for the $1,000 \mathrm{~Hz}$ sampling rate. These values are close to that of the reference step response which indicates that the proposed method designed by the model reference approach can achieve a good tracking performance in relation to the reference output at different sampling frequencies. In contrast with the output tracking integrated discrete PID-based SMC designed by the traditional design approach, less overshoot was observed in the step response because the derivability of the reference signal is not required in the model reference approach.

\subsection{Dynamic Tracking Performance Compared with the Continuous PID-based SMC}

In order to verify the effectiveness of proposed control method, dynamic tracking experiments with the above sampling frequencies were also carried out on the PEA. Particularly, three types of inputs were used as the reference signal: (1) the sinusoidal inputs of an amplitude of $5 \mu \mathrm{m}$ with different frequencies of 1,10 , 30 and $50 \mathrm{~Hz}$; (2) the piecewise continuous combination of different-amplitude sinusoidal inputs with the same frequency (PSWS) [13]; (3) the superposition of four sinusoidal inputs with different frequencies, amplitudes and phase delays (SW) [13].

The output tracking integrated discrete PID-based SMC designed by the model reference approach was implemented on the PEA, in which the parameters of the second order reference model were chosen to be $\varsigma=0.25$ and $\omega_{n}=3,000 \mathrm{rad} / \mathrm{s}$. For comparison, the 
continuous PID-based SMC introduced in Ref. [7] was also implemented on the PEA. The parameters of the PID regulator were adjusted by using the Ziegler-Nichols method, leading to $P=0.001, I=1$, and $D=0.0000001$ for the $20,000 \mathrm{~Hz}$ sampling rate, $P$ $=0.002, I=0.6$, and $D=0.0000001$ for the $10,000 \mathrm{~Hz}$ sampling rate, and $P=0.0001, I=0.03$, and $\mathrm{D}=$ 0.0000001 for the $5,000 \mathrm{~Hz}$ sampling rate.

Tables 1 and 2 show the dynamic tracking performance of the output tracking integrated discrete PID-based SMC designed by the model reference approach at 5,000 $\mathrm{Hz}$ sampling frequency, compared with the continuous PID-based SMC. The tracking error was calculated in terms of the 2-norm of the difference between the desired output and the measured output.

It can be concluded from the comparison that the output tracking integrated discrete PID-based SMC performs similar as the continuous PID-based SMC at high sampling frequencies. For example, the tracking error for $1 \mathrm{~Hz} 5 \mu \mathrm{m}$ sinusoidal input controlled by the discrete PID-based SMC developed from the model reference approach is $0.0088 \mu \mathrm{m}$ which is only 0.0026 $\mu \mathrm{m}$ more than that controlled by the continuous PID-based SMC, while for $50 \mathrm{~Hz}$ sinusoidal input, the tracking error is $0.0363 \mu \mathrm{m}$ less than that of the continuous PID-based SMC. As sampling frequency decreases, the output tracking integrated discrete PID-based SMC performs much better than the continuous PID-based SMC with one exception at a frequency of $1 \mathrm{~Hz}$, which is bolded in Table 2. In addition, the performance improvement with the proposed method is not apparent at low input frequency. However, as frequency increases, the improvement becomes more profound.

It is noted that as compared with the traditional SMC design approach, the model reference approach can achieve better tracking performance. For example, for the $30 \mathrm{~Hz}$ sinusoid reference input with $20,000 \mathrm{~Hz}$ sampling rate, the tracking error by using the model reference approach is $0.1253-0.0301 \mu \mathrm{m}$ less than that
Table 1 Comparison of the tracking performance between the proposed method and the continuous PID-based SMC at $20,000 \mathrm{~Hz}$ sampling rate. (A: discrete PID-based SMC with traditional design approach; B: Discrete PID-based SMC with model reference approach; $\mathrm{C}$ : Continuous PID-based SMC).

\begin{tabular}{llll}
\hline Reference inputs & A & B & C \\
\hline 1 Hz sinusoidal input & 0.0117 & 0.0088 & 0.0062 \\
10 Hz sinusoidal input & 0.0519 & 0.0383 & 0.0213 \\
30 Hz sinusoidal input & 0.1552 & 0.1253 & 0.1001 \\
$50 \mathrm{~Hz}$ sinusoidal input & 0.2641 & 0.2315 & 0.2678 \\
SW with $f_{\max }=10 \mathrm{~Hz}$ & 0.0142 & 0.0106 & 0.0074 \\
SW with $f_{\max }=30 \mathrm{~Hz}$ & 0.0223 & 0.0188 & 0.0169 \\
SW with $f_{\max }=50 \mathrm{~Hz}$ & 0.0313 & 0.0309 & 0.0352 \\
PSWS with $10 \mathrm{~Hz}$ frequency & 0.0393 & 0.0295 & 0.0247 \\
PSWS with $30 \mathrm{~Hz}$ frequency & 0.1188 & 0.0964 & 0.0979 \\
PSWS with $50 \mathrm{~Hz}$ frequency & 0.2000 & 0.1769 & 0.1833 \\
\hline
\end{tabular}

Table 2 Comparison of the tracking performance between the proposed method and the continuous PID-based SMC at $5,000 \mathrm{~Hz}$ sampling rate. (Same definition of $A, B, C$ as Table 1).

\begin{tabular}{llll}
\hline Reference inputs & A & B & C \\
\hline 1 Hz sinusoidal input & 0.0511 & 0.0283 & 0.0121 \\
10 Hz sinusoidal input & 0.0620 & 0.0385 & 0.1535 \\
30 Hz sinusoidal input & 0.1600 & 0.0833 & 1.2270 \\
$50 \mathrm{~Hz}$ sinusoidal input & 0.3685 & 0.1865 & 2.9360 \\
SW with $f_{\max }=10 \mathrm{~Hz}$ & 0.0372 & 0.0218 & 0.0479 \\
SW with $f_{\max }=30 \mathrm{~Hz}$ & 0.0635 & 0.0400 & 0.1976 \\
SW with $f_{\max }=50 \mathrm{~Hz}$ & 0.0849 & 0.0541 & 0.6206 \\
PSWS with $10 \mathrm{~Hz}$ frequency & 0.0617 & 0.0384 & 0.1057 \\
PSWS with $30 \mathrm{~Hz}$ frequency & 0.1423 & 0.0812 & 0.8469 \\
PSWS with $50 \mathrm{~Hz}$ frequency & 0.2835 & 0.1430 & 2.0464 \\
\hline
\end{tabular}

obtained by using the traditional SMC design approach. Moreover, increasing the sampling rate improves the tracking performance of discrete PID-based SMC for both the traditional SMC design approach and the model reference approach.

\subsection{Dynamic Tracking under Different Sampling Rates}

The improvement of tracking performance with the output tracking integrated discrete PID-based SMC can be further illustrated by experiments with varying sampling rates. Specifically, sinusoidal tracking experiments were implemented on the PEA at sampling rate of $1,000 \mathrm{~Hz}, 2,000 \mathrm{~Hz}, 5,000 \mathrm{~Hz}, 10,000$ 


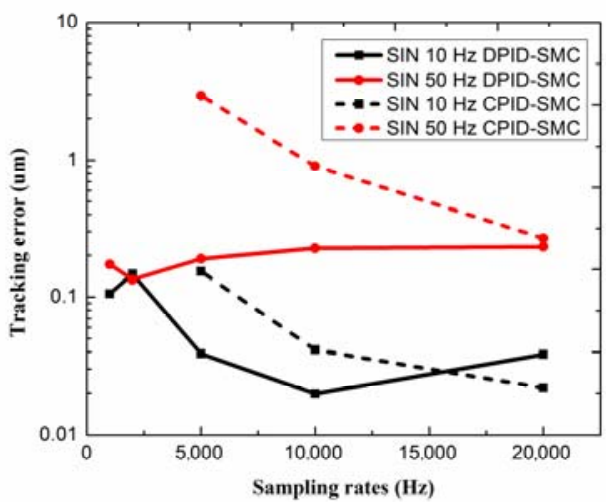

Fig. 3 Comparison of tracking errors, as controlled by the discrete and continuous PID-based SMC with different sampling rates (tracking errors for the continuous PID-based SMC at $1,000 \mathrm{~Hz}$ and $2,000 \mathrm{~Hz}$ are not given as they are significantly large).

$\mathrm{Hz}$, and 20,000 Hz, respectively. Both the discrete PID-based SMCs, as designed by the model reference approach and the continuous PID-based SMC approach, were used to control the PEA. The parameters of the continuous PID-based SMC were adjusted to be $P=$ $0.002, I=0.6, D=0.0000001$ for the case with the $10,000 \mathrm{~Hz}$ sampling rate. Fig. 3 shows the tracking error varying with the sampling rates by using these two controllers. It can be seen that the discrete PID-based SMC performs better than the continuous one, especially at low sampling rates.

\section{Conclusions}

This paper presents the development of the output tracking integrated discrete PID-based SMC for SISO system, in which the system output is fed back for generating the control action. As such, it can be employed in the applications where the system states cannot be readily available. Both the traditional SMC design approach and the model reference approach are employed for the controller design. By applying the PID regulator instead of the 'bang-bang' switching control, chattering, and the zigzag state motion thus the steady state error, were eliminated. In order to verify the effectiveness of the proposed control schemes, experiments were carried out on a commercially available PEA with varying sampling frequencies, as compared to the use of continuous PID-based SMC. The results show that the developed method as designed by both approaches can achieve better tracking performance and that as the input frequency increases; the performance improvement with the developed method becomes more profound. The results also show that due to the non-requirement on the derivability of the reference signal, the developed method designed by the model reference approach has a better performance than the one designed by the traditional SMC design approach.

\section{References}

[1] Young, K. D., Utkin, V. I., and Özgüner, U. 1999. “A Control Engineer's Guide to Sliding Mode Control.” IEEE Transactions on Control Systems Technology 7 (3): 328-42.

[2] Edwards, C., and Spurgeon, S. K. 1998. Sliding Mode Control. London: Taylor \& Francis Ltd.

[3] Liawa, H. C., Shirinzadeh, B., and Smith, J. 2007. "Enhanced Sliding Mode Motion Tracking Control of Piezoelectric Actuators." Sensors and Actuators A: Physical 138 (1): 194-202.

[4] Wang, S., Habibi, S., and Burton, R., "Sliding Mode Control for an Electrohydraulic Actuator System with Discontinuous non-linear Friction." In Proceedings of the Institute of Mechanical Engineers, Part I: Journal of Systems and Control Engineering, 799-815.

[5] Levant, A. 2005. "Homogeneity Approach to High-Order Sliding Mode Design.” Automatica 41: 823-30.

[6] Fridman, L., and Levant, A. 2002. "Higher Order Sliding Modes." In Sliding Mode Control in Engineering, edited by Perruquetti, W., and Barbot, J. P. New York: Marcel Dekker.

[7] Peng, J. Y., and Chen, X. B. 2011. "Integrated PID-based Sliding Mode State Estimation and Control for Piezoelectric Actuators." IEEE/ASME Transactions on Mechatronics 19 (1) doi:10.1109/TMECH.2012.2222428.

[8] Nise, N. S. 2011. Control System Engineering. 6th edition. New Jersey: John Wiley \& Sons, Inc.

[9] Gao, W. B., Wang, Y. F., and Homaifa, A. 1995. "Discrete-Time Variable Structure Control Systems." IEEE Transactions on Industrial Electronics 42 (2): 117-22.

[10] Bartoszewicz, A. 1998. "Discrete-Sliding Mode Control Strategies." IEEE Transactions on Industrial Electronics 45 (4): 627-33. 
[11] Lin, Y., Shi. Y., and Burton, R. 2013. "Modeling and Robust Discrete-Time Sliding Mode Control Design for a Fluid Power Electrohydraulic Actuator System." IEEE/ASME Transactions on Mechatronics 18 (1): $1-10$.

[12] Chang, J. L. 2008. "Combing State Estimator and Disturbance Observer in Discrete-time Sliding Mode Controller Design." Asian Journal of Control 10 (5): 515-24.

[13] Cao, Y., and Chen, X. B. 2012, "Integrated Inversion-Feedforward and PID-based Sliding Mode Control for Piezoelectric Actuators." Presented at the American Control Conference, Montreal, Canada.

[14] Cao, Y., and Chen, X. B. 2014. "An Output Tracking based Discrete PID-Sliding Mode Control on MIMO systems." IEEE/ASME Transactions on Mechatroics 19
(4): 1183-94

[15] Corft, D., Shed, G., and Devasia, S. 2001. "Creep, Hysteresis, and Vibration Compensation for Piezoactuators: Atomic Force Microscopy Application." Journal of Dynamic Systems Measurement and Control-Transactions of the ASME 123 (1): 35-43.

[16] Devasia, S., and Moheimani, S. O. R. 2007. "A Survey of Control Issues in Nano-positioning." IEEE Transactions on Control System Technology 15 (5): 802-23.

[17] Cao, Y., and Chen, X. B. 2012. "A Novel Discrete ARMA-based Model for Piezoelectric Actuator Hysteresis." IEEE Transactions on Mechatronics 17 (4): 737-44

[18] Chen, X. B., Zhang, Q. S., Kang, D., and Zhang, W. J. 2008. "On the Dynamics of Piezoelectric Positioning Systems.” Review of Scientific Instrument 79. 116101. 